\title{
INVITED: Linear Periodically Time-Varying (LPTV) Circuits Enable New Radio Architectures for Emerging Wireless Communication Paradigms
}

\author{
Extended Abstract
}

\author{
Negar Reiskarimian \\ Columbia University \\ 1300 SW Mudd, 500 W 120th St., \\ New York, NY 10027 \\ nr2475@columbia.edu
}

\author{
Linxiao Zhang \\ Columbia University \\ 1300 SW Mudd, 500 W 120th St., \\ New York, NY 10027 \\ lz2307@columbia.edu
}

\author{
Harish Krishnaswamy \\ Columbia University \\ 1300 SW Mudd, 500 W 120th St., \\ New York, NY 10027 \\ harish@ee.columbia.edu
}

\begin{abstract}
The next generation of cellular wireless communication networks (the much hyped " $5 \mathrm{G}$ ") is targeting a $1000 \mathrm{x}$ increase in data capacity. This has sparked an investigation of new and transformative wireless communication paradigms, including massive MIMO, full duplex and millimeter-wave wireless. These new wireless paradigms place requirements on the radio circuitry that are orders of magnitude more challenging than traditional systems, forcing us to rethink conventional radio design. Conventional analog and radio frequency circuit design has relied on linear, time-invariant (LTI) components and circuits. However, LTI components and circuits are restricted in the signal processing functionalities that can be implemented. Recently, there has been significant interest in linear, periodically time varying (LPTV) circuits that can enable new functionalities and components, such as highly-tunable, high quality integrated filters, front-ends with spatio-spectral filtering capability and integrated non-magnetic non-reciprocal components such as circulators and isolators. This paper reviews recent research breakthroughs in LPTV circuits and systems that enable full-duplex and massive MIMO wireless.
\end{abstract}

\section{CCS CONCEPTS}

- Hardware $\rightarrow$ Wireless devices; Radio frequency and wireless circuits;

\section{KEYWORDS}

Linear Periodically Time-Varying; LPTV; Wireless Communication; MIMO; Full-Duplex; Circulator; RFIC

Permission to make digital or hard copies of all or part of this work for personal or classroom use is granted without fee provided that copies are not made or distributed for profit or commercial advantage and that copies bear this notice and the full citation on the first page. Copyrights for components of this work owned by others than ACM must be honored. Abstracting with credit is permitted. To copy otherwise, or republish, to post on servers or to redistribute to lists, requires prior specific permission and/or a fee. Request permissions from Permissions@acm.org. DAC '17, June 18-22, 2017, Austin, TX, USA

(C) 2017 Association for Computing Machinery.

ACM ISBN 978-1-4503-4927-7/17/06 ..\$15.00

http://dx.doi.org/10.1145/3061639.3072954

\section{INTRODUCTION}

The $5 \mathrm{G} 1000 \mathrm{x}$ data capacity challenge over the next ten years is driven by the ever-increasing reliance of society on wireless communications, and the consequent near-doubling of wireless data throughput every year. This $1000 x$ increase in data capacity is expected to be met through three means - densification, increased spectral efficiency and increased bandwidth through the exploration of new spectrum. Densification, or the reduction in cell size through the denser deployment of small-cell base stations, will enable provision of service to more mobile devices within a given area, thus increasing aggregate network throughput. Increased spectral efficiency can be achieved through new communication paradigms such as massive MIMO [1] and full duplex [2]. Massive MIMO involves the use of an extremely large number of antennas at the base station to simultaneously serve multiple users within the same spectrum (often more precisely called MU-massive-MIMO or multi-user massive MIMO), thus greatly enhancing spectral efficiency. Full duplex wireless involves simultaneous transmission and reception on the same frequency, thus doubling spectral efficiency immediately in the physical layer, while offering numerous other benefits at the higher layers. Finally, wider bandwidths can supported by migrating up into the millimeter-wave regime $(30 \mathrm{GHz}$ and beyond) where wide swaths of spectrum are available. Currently, frequency bands at $28 \mathrm{GHz}$ and $38 \mathrm{GHz}$ are being strongly considered for $5 \mathrm{G}$, among others [3].

However, these new wireless communication paradigms significantly challenge the radio hardware. For instance, full duplex operation requires the receiver to operate in the presence of significant self-interference from its own transmitter. This selfinterference can be a billion to a trillion times more powerful than the desired signal, requiring self-interference or echo cancellation circuitry and algorithms that must function with one-part-perbillion/trillion accuracy. Massive MIMO requires the integration of hundreds of radio chains, each of which must consequently be extremely power efficient while maintaining high dynamic range to tolerate unknown external interference.

Traditional radio circuit design has relied on conventional linear, time-invariant (LTI) circuits and systems, but such design has plateaued in terms of achievable performance and functionality. In 

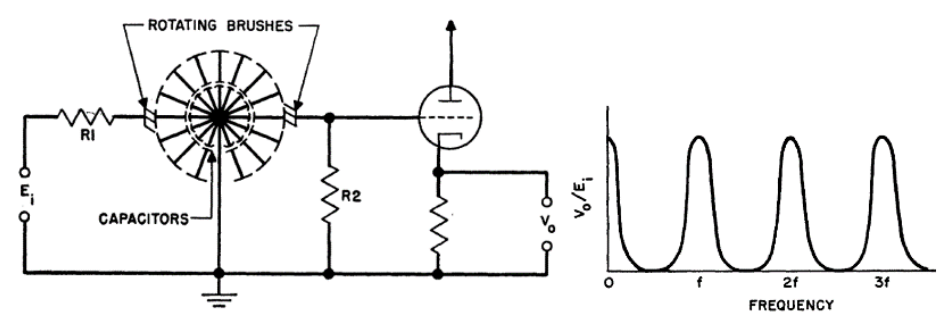

(a)
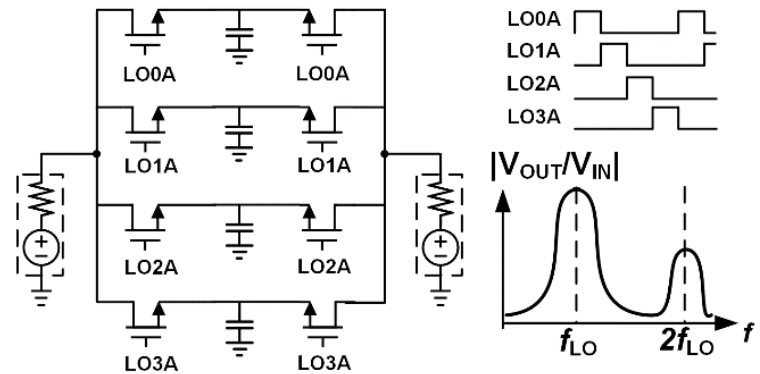

(b)

Figure 1: Commutated networks then and now: (a) Electro-mechanical implementation of comb filter through capacitive commutation [4]. (b) Modern CMOS implementation of the N-path filter.

the last few years, we have seen very exciting research progress on linear, periodically time varying (LPTV) circuits and systems, which enable new functionalities that are otherwise unachievable. Examples of these functionalities include highly-tunable, high quality integrated filters (the so-called "N-path filters"), frontends with spatio-spectral filtering capability for (massive) MIMO and integrated non-magnetic non-reciprocal components such as circulators that enable full duplex wireless. This paper reviews recent research breakthroughs in LPTV circuits and systems that enable new wireless communication paradigms.

\section{THE REVIVAL OF THE N-PATH FILTER}

The N-path filter is a commutated network, essentially a class of LPTV networks where the signal is periodically commutated through a bank of LTI networks. The first commutated networks relied on mechanical commutation through a rotating brush that periodically contacted a bank of capacitors to realize narrow comb filters around harmonics of the commutation frequency (Fig. 1(a)) [4]. More recently, electronic commutation using passive transistor-based switches has resulted in high quality-factor (Q) comb filters, commonly called N-path filters, which operate at RF, exhibit significantly lower noise and higher linearity when compared with active RF filters, are compatible and scale well with CMOS IC technology, and exhibit the potential to replace the traditional front-end off-chip surface acoustic wave (SAW) filters that are widely used in communication applications. Aside from the high-Q, low noise and high linearity, the center frequency of the filter is defined by the commutation clock frequency, implying that the filter can be widely tuned over decades of frequency by tuning the clock frequency. This is in stark contrast to SAW filters, which are fixed in frequency, necessitating a large array of SAW filters for each frequency band used in today's 4G cellular networks. Over the past several years, there has been extensive research on N-path filters in the CMOS RFIC community, and this research has enabled the development of SAW-less receivers for cellular communication applications [5-7].

\section{APPLICATION OF N-PATH FILTER CONCEPTS TO SPATIO-SPECTRAL FILTERING IN MIMO ARRAYS}

The inherent principle behind N-path filters is that passive transistor-based switches, or passive mixers, are able to translate impedances at baseband frequencies to RF around the switching or commutation frequency. In the basic N-path filter configuration, the low-pass filtering profile created by the capacitors is translated to RF to realize a second-order bandpass filter (Fig. 1(b)). In this section, we describe how this impedance translation principle can be exploited for MIMO receiver applications.

As mentioned earlier, massive MIMO wireless has drawn tremendous interest for $5 \mathrm{G}$ applications. MIMO signal processing can practically only be implemented in the digital domain, and therefore MIMO receivers typically employ digitization at every element. In other words, each antenna employs a dedicated receiver chain with down-conversion mixers and analog-to-digital converters (ADCs). Such digital MIMO receivers can also exploit digital signal processing (DSP) for digital array calibration, which is crucial for complex, large-scale arrays.

However, the digital MIMO receiver architecture has a major disadvantage, namely that spatial selectivity is missing in the $\mathrm{RF} /$ analog domain. At the target frequency band, multiple spatial signals can arrive at the antenna array at different power levels. The instantaneous dynamic range required for the RF/analog circuits and the ADCs will be limited by the strongest spatial signal on the upper bound, and the weakest spatial signal on the lower bound. A high instantaneous dynamic range requirement directly translates to high power consumption and high cost.

Recently, we developed a scalable spatial notch suppression technique towards recovering $\mathrm{RF} /$ analog spatial selectivity in a digital MIMO receiver. Knowing the direction of a strong spatial blocker, a spatial notch, instead of beams, can be synthesized in the blocker direction to filter it out. As a result, all the analog baseband outputs will show high conversion gains to signals from all directions except the blocker direction.

In order to synthesize the scalable spatial notch in the baseband, a spatial notch filter (SNF) is designed to provide lower input impedance in the blocker direction and high impedance in other directions. This is achieved by connecting operational transconductance amplifiers (OTAs) with resistive feedback from each element to a common node. When signals are incident on the 


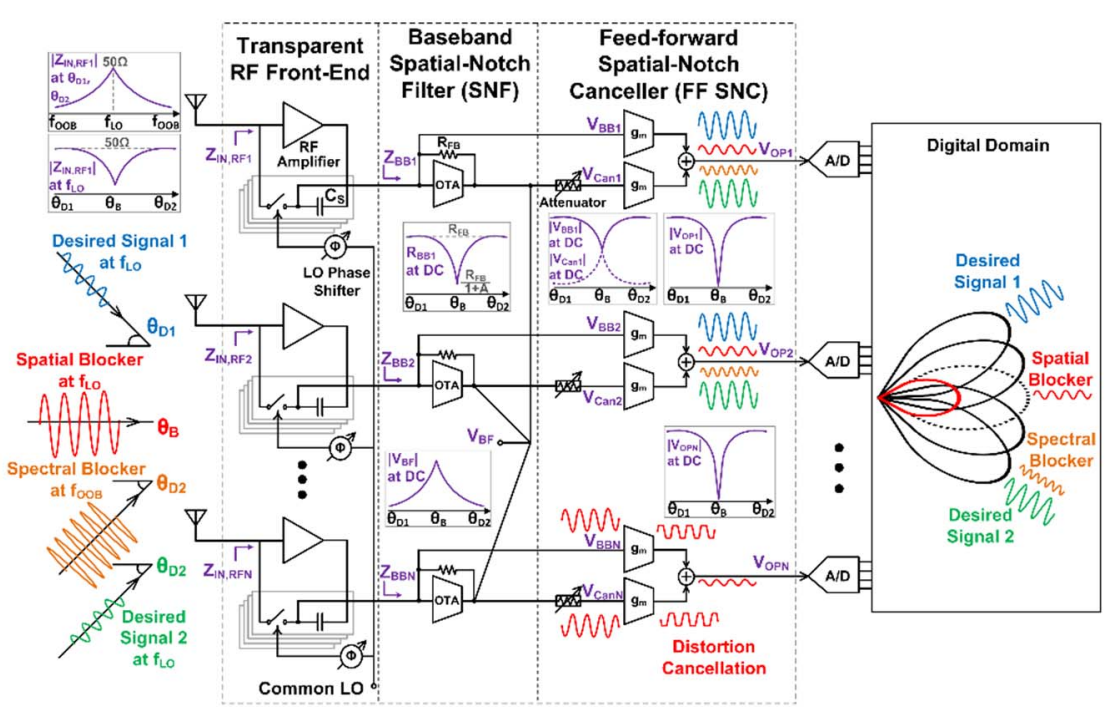

(a)

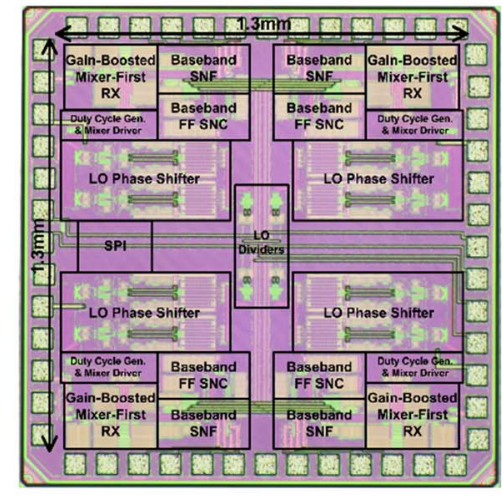

(b)

Figure 2: (a) Block diagram of a 0.1-1.7GHz 65nm CMOS 4-element MIMO receiver array with analog/RF spatial notch suppression. (b) Chip microphotograph.

OTAs with identical magnitude and phase (i.e. the blocker direction), each OTA presents high gain and consequently a low input impedance through the feedback resistor. When signals are incident with unequal phases (i.e. away from the blocker direction), the OTAs experience destructive interference at their outputs and hence low gain. Consequently, high input impedance is seen through the feedback resistance. Using this spatially modulated impedance to load a current mode receiver leads to spatially modulated conversion gain. A gain-boosted passivemixer-first receiver translates this impedance to the antenna interface to achieve spatial notch suppression right at the antennas. Thus, the impedance translation property of LPTV passive mixers enables spatial filtering early in the receiver chain. The LPTV passive mixers also exhibit high-Q tunable frequencydomain filtering as described before, and therefore, the overall receiver features both spatial and spectral filtering capability. The spatial notch suppression is scalable through the common OTA output node. The tiling of multiple ICs on the same PCB with their common OTA output node connected enables the realization of larger MIMO arrays with scaled spatial notch suppression.

A prototype receiver array was implemented in a $65 \mathrm{~nm}$ CMOS process (Fig. 2). Experimental results showed $32 \mathrm{~dB}$ steerable spatial notch suppression, and scalability across two ICs. A wireless multi-beam imaging demonstration was conducted in the presence of a strong spatial blocker to demonstrate the spatial filtering functionality. The reader is directed to [8-10] for additional details.

\section{APPLICATION OF N-PATH FILTERS TO INTEGRATED NON-MAGNETIC NON- RECIPROCAL COMPONENTS}

Another exciting property of LPTV networks is the ability to break reciprocity. Linear, time-invariant, passive circuits and systems constructed from conventional materials are reciprocal. Reciprocity places fundamental restrictions on the types of functionalities and components that can be implemented. For instance, for full-duplex wireless, it is desirable for the transmitter and the receiver to share the same antenna while transmitting and receiving at the same time at the same frequency. While twoantenna full-duplex wireless transceivers are possible, such solutions are bulky and do not translate well to MIMO and diversity applications. Non-reciprocal circulators, which route the transmitter signal to the antenna, but route the antenna signal to the receiver, enable full duplex on a single antenna.

Traditionally, magnetic materials such as ferrites have been the basis of implementing non-reciprocal elements. Ferrite materials lose reciprocity under the application of an external magnetic field through the Faraday Effect. However non-reciprocal components based on magnetic materials are bulky, expensive and not compatible with CMOS integration. Other approaches of enabling non-reciprocity include the use active devices, which are limited in noise and linearity performance, and non-linear devices, which are limited in the range of signal powers over which they operate. More recently, LPTV circuits have also been shown to enable non-magnetic non-reciprocity through time-varying permittivity modulation $[11,12]$. However, permittivity modulation in CMOS is limited in the modulation index $\left(\mathrm{C}_{\mathrm{on}} / \mathrm{C}_{\text {off }}\right)$ achievable in varactors, resulting in nonreciprocal components that are either bulky or lossy.

We have found that applying a relative phase shift to the nonoverlapping clocks driving the input and output switch sets of a two-port N-path filter imparts a nonreciprocal phase-shift to the signals traveling in the forward and reverse directions since they see a different ordering of the phase-shifted switches. The magnitude response remains reciprocal and low-loss, similar to traditional N-path filters (Fig. 3(a)). To convert phase nonreciprocity to non-reciprocal wave propagation, an N-path-filter with $+/-90^{\circ}$ phase-shift is placed inside a transmission line loop 


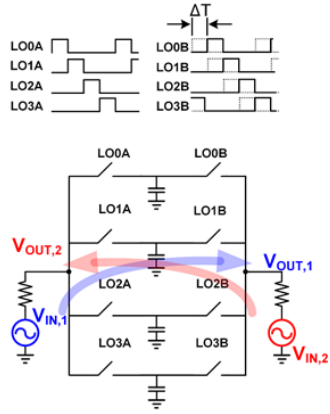

(a)

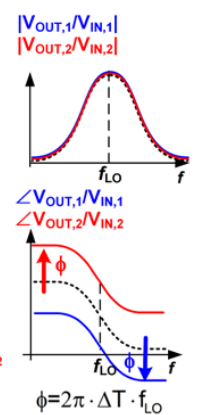

$\phi=2 \pi \cdot \Delta \mathrm{T} \cdot \mathrm{f}_{\mathrm{L}}$

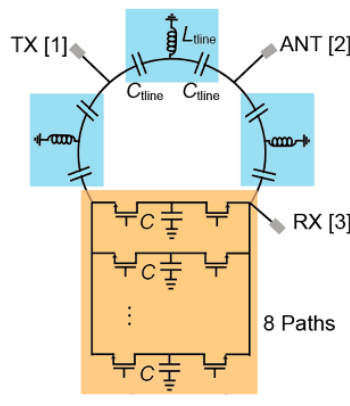

(b)

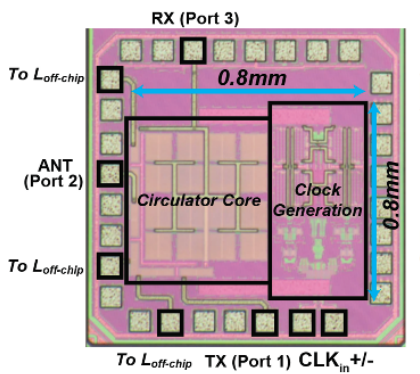

(c)

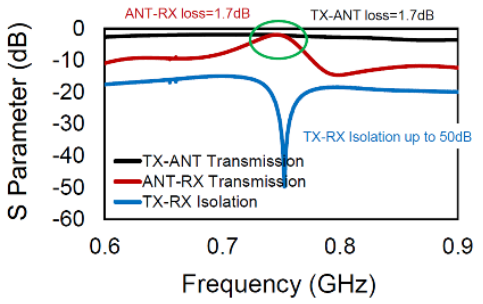

(d)

Figure 3: Integrated non-magnetic circulator: (a) non-reciprocity induced by phase-shifted N-path commutation, (b) 3-port circulator structure obtained by placing the non-reciprocal two-port N-path filter with $90^{\circ}$ phase-shift within a miniaturized $3 \lambda / 4$ transmission line loop (using three lumped CLC sections). (d) Chip microphotograph of the circulator (inductors placed off-chip). (e) Circulator S-parameter measurements.

with a length of $3 \lambda / 4$ (Fig. 3(b)). The combination of the nonreciprocal phase shift of the N-path filter with the reciprocal phase shift of the transmission line results in unidirectional wave propagation $\left(-270^{\circ}-90^{\circ}=-360^{\circ}\right)$, because the boundary condition for wave propagation in the reverse direction cannot be satisfied ($\left.270^{\circ}+90^{\circ}=-180^{\circ}\right)$. Additionally, a three-port circulator can be realized by placing ports anywhere along the loop as long as they maintain a $N / 4$ circumferential distance between them.

A prototype circulator operating over $610-850 \mathrm{MHz}$ was implemented in a $65 \mathrm{~nm}$ CMOS process (Fig. 3(c)). This represented the first non-magnetic non-reciprocal circulator implemented in CMOS. The reader is referred to [13] for additional details. We have also designed and fabricated a fullduplex receiver in $65 \mathrm{~nm}$ CMOS using our circulator as the sharedantenna interface that can handle up to $-7 \mathrm{dBm}$ of transmitted power $[14,15]$. The circulator serves as a first stage of selfinterference suppression by providing TX-RX isolation. The fullduplex receiver has an additional baseband self-interference canceller to relax the analog to digital converter (ADC) dynamic range. Measurements reveal $42 \mathrm{~dB}$ of self-interference cancellation across the circulator and analog baseband canceller through a joint optimization over a bandwidth of $12 \mathrm{MHz}$. Digital SIC has also been implemented in Matlab after capturing the BB signals using an oscilloscope to achieve an overall $85 \mathrm{~dB}$ self-interference suppression across the FD receiver. More recent advances include a combined circulator-receiver for full duplex that combines the circulator with down-conversion functionality [16], enabling higher transmitter power handling, lower power consumption and better noise performance, and an extension of the circulator to millimeter-waves $(28 \mathrm{GHz})$ [17].

\section{CONCLUSIONS}

LTPV circuits and systems have reinvigorated analog/RF circuit design over the past five to ten years, and have enabled new circuits, components and architectures for radios targeting new wireless communication paradigms, such as massive MIMO and full-duplex. Topics for the future include scaling LPTV circuits to millimeter-wave, enabling the combination of millimeter-wave operation with paradigms such as full-duplex and massive MIMO.

\section{REFERENCES}

[1] E. G. Larsson, O. Edfors, F. Tufvesson, and T. L. Marzetta, "Massive MIMO for next generation wireless systems," IEEE Communications Magazine, vol. 52, no. 2, pp. 186-195, Feb 2014.

[2] D. Bharadia, E. McMilin, and S. Katti, "Full duplex radios," in Proc. ACM SIGCOMM, 2013, pp. 375-386.

[3] Theodore S. Rappaport et al. "Millimeter wave mobile communications for 5G cellular: It will work!." IEEE Access, vol. 1, pp. 335-349, 2013.

[4] H. Busignies and M. Dishal, "Some Relations between Speed of Indication, Bandwidth, and Signal-to-Random-Noise Ratio in Radio Navigation and Direction Finding," in Proceedings of the IRE, vol. 37, no. 5, pp. 478-488, May 1949.

[5] A.Ghaffari, E.Klumperink, M.Soer, and B.Nauta, "Tunable high-Q N-path band-pass filters: Modeling and verification," IEEE Journal of Solid-State Circuits, vol. 46, no. 5, pp. 998-1010, May 2011.

[6] C. Andrews and A. C. Molnar, "A passive mixer-first receiver with digitally controlled and widely tunable RF interface," IEEE Journal of Solid-State Circuits, vol. 45, no. 12, pp. 2696-2708, Dec. 2010.

[7] N. Reiskarimian and H. Krishnaswamy, "Design of all-passive higher-order CMOS N-path filters." in 2015 IEEE Radio Frequency Integrated Circuits Symposium, pp. 83-86, 2015.

[8] L. Zhang, A. Natarajan and H. Krishnaswamy, "A scalable 0.1-to-1.7GHz spatio-spectral-filtering 4-element MIMO receiver array with spatial notch suppression enabling digital beamforming," in 2016 IEEE International SolidState Circuits Conference (ISSCC), pp. 166-167, Feb. 2016.

[9] H. Krishnaswamy and L. Zhang, "Analog and RF Interference Mitigation for Integrated MIMO Receiver Arrays," Proceedings of the IEEE, vol. 104 , no. 3, pp. 561-575, March 2016

[10] L. Zhang, A. Natarajan, and H. Krishnaswamy, "Scalable Spatial Notch Suppression in Spatio-Spectral-Filtering MIMO Receiver Arrays for Digital Beamforming," IEEE Journal of Solid-State Circuits, vol. 51, no. 12, pp. 3152 3166, Dec 2016.

[11] S. Qin, Q. Xu and Y. E. Wang, "Nonreciprocal Components With Distributedly Modulated Capacitors," in IEEE Transactions on Microwave Theory and Techniques, vol. 62, no. 10, pp. 2260-2272, Oct. 2014.

[12] N. A. Estep, D. L. Sounas, J. Soric and A. Alù, "Magnetic-free non-reciprocity and isolation based on parametrically modulated coupled-resonator loops," Nature Physics, vol. 10, no. 11, pp 923-927, November 2014.

[13] N. Reiskarimian and H. Krishnaswamy, "Magnetic-Free Non-Reciprocity Based on Staggered Commutation," Nature Communications, vol. 7, no. 4, April 2016.

[14] J. Zhou, N. Reiskarimian and H. Krishnaswamy, "Receiver with integrated magnetic-free N-path-filter-based non-reciprocal circulator and baseband selfinterference cancellation for full-duplex wireless," 2016 IEEE International Solid-State Circuits Conference (ISSCC), pp. 178-180, Feb. 2016.

[15] N. Reiskarimian, J. Zhou and H. Krishnaswamy, "A CMOS Passive LPTV Nonmagnetic Circulator and Its Application in a Full-Duplex Receiver," accepted and to appear in the IEEE Journal of Solid-State Circuits, 2017.

[16] N. Reiskarimian, M. Baraani Dastjerdi, J. Zhou and H. Krishnaswamy, "Highly-linear integrated magnetic-free circulator-receiver for full-duplex wireless," 2017 IEEE International Solid-State Circuits Conference (ISSCC), pp. 316-317, Feb. 2017

[17] T. Dinc and H. Krishnaswamy, "A $28 \mathrm{GHz}$ Magnetic-Free Non-reciprocal Passive CMOS Circulator Based on Spatio-Temporal Conductance Modulation", in the 2017 IEEE International Solid-State Circuits Conference (ISSCC), pp. 294-295, Feb. 2017. 